\title{
Paroxetine improves social anxiety disorder in children and adolescents
}

Wagner KD, Berard R, Stein MB, et al. A multicenter, randomized, double-blind, placebo-controlled trial of paroxetine in children and adolescents with social anxiety disorder. Arch Gen Psychiatry 2004;61:1153-62.

Is paroxetine effective and well tolerated in children and adolescents with social anxiety disorder?

\section{METHODS}

L

Design: Randomised controlled trial.

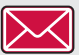

Allocation: Concealed.

Blinding: Double blinded

I

Follow up period: 16 weeks.

Setting: 38 centres in the USA, South Africa, Canada, and Belgium; enrolment November 1999 to October 2001.

Patients: 322 children and adolescents (8-17 years) with social anxiety disorder (DSM-IV). Exclusions: other axis I disorder; experiencing a major depressive episode or suicidal tendencies; history of bipolar disorder, psychosis, developmental disorder, or serious medical condition; receiving psychoactive medication or therapy; recent electroconvulsive therapy; substance abuse; hypersensitivity to serotonin selective reuptake inhibitors (SSRIs) pregnant or lactating; or abnormal laboratory or ECG results.

D Intervention: Paroxetine $(10 \mathrm{mg} /$ day for the first week, increased by $10 \mathrm{mg} /$ week up to a maximum dose of $50 \mathrm{mg} /$ day); placebo. All participants received age appropriate psychoeducational pamphlets on social anxiety disorder and suggestions for self-help. Parents/guardians also received a pamphlet that included this information, as well as reasons for disorder development, symptoms, and what they could do to help.

Outcomes: Response ("very much improved" or "much improved" on the Clinical Global Impression-Improvement Scale); adverse events.

\section{MAIN RESULTS}

Paroxetine significantly increased the proportion of responders (125/ $161(78 \%))$ at 16 weeks, compared with placebo (59/154 (38\%)) (adjusted OR 7, 95\% CI 4.1 to 12.1 ). Average paroxetine dose was $26.5 \mathrm{mg} /$ day for children and $35 \mathrm{mg}$ /day for adolescents at 16 weeks. The commonest adverse effects were insomnia, decreased appetite, and vomiting (paroxetine $v$ placebo: insomnia $14 \% \vee 6 \%, \mathrm{p}=0.02$; decreased appetite $8 \% \vee 3 \%, \mathrm{p}=0.11$; vomiting $7 \% \vee 2 \%, \mathrm{p}=0.07$ ). In the paroxetine group five had suicidal ideation, threatened suicide, or self harmed, compared with none in the placebo group $(\mathrm{p}=0.06)$. Rate of withdrawal due to adverse events was low (paroxetine $v$ placebo: $5.5 \% \vee 1.3 \%$, p value not reported). More people withdrew from the placebo group (paroxetine $v$ placebo: $24 \% v 33 \%$ ).

For correspondence: Dr Karen Dineen Wagner, Division of Child and Adolescent Psychiatry, Department of Psychiatry and Behavioural Sciences, University of Texas Medical Branch, 301 University Boulevard, Galveston, TX 77555-0188, USA; kwagner@utmb.edu

Sources of funding: GlaxoSmithKline, USA.

\section{CONCLUSIONS}

Paroxetine is effective for young people and adolescents with social anxiety disorder and produces few serious adverse effects. Response rate is higher than previously reported for paroxetine in adults with social anxiety disorder.

\section{NOTES}

The authors intended to use last observation carried forward analysis for people with at least one follow up assessment (99\% participants; 163 in paroxetine group, 156 in placebo group). It is unclear why the denominator used in the response analysis differed from these figures.

\section{Commentary}

A nxiety disorders are highly prevalent and often debilitating conditions in childhood and adolescence. 'Many randomised controlled trials (RCTs) support cognitive behavioural therapy (CBT) as an effective treatment, but samples have typically consisted of mild to moderately ill children. ${ }^{2}$ Serotonin selective reuptake inhibitors (SSRIs) are also frequently used, especially in more severely affected children, but the evidence for their use is limited. A single multisite RCT in children has demonstrated the efficacy of fluvoxamine for various childhood anxiety disorders. ${ }^{3}$ Recent concerns about possible medication related suicidal ideation may dampen clinicians' enthusiasm for these potentially valuable medications. Further well controlled trials using SSRIs in anxious children are therefore sorely needed.

Wagner et al's industry sponsored trial, demonstrating efficacy and tolerability of paroxetine for social anxiety in children and adolescents, has several methodological strengths. As anxiety disorders in children often co-occur, the authors have included subjects with anxious comorbidity, improving generalisability. However, children with comorbid mood or substance use disorders and those receiving psychotherapy were excluded. The authors analysed an "intent to treat" sample (not just study completers), thus accounting for dropout due to side effects, lack of efficacy, or other causes. They noted interesting age related differences in side effects, with nervousness and hyperkinesia commoner in younger children than adolescents.

Based on these findings the clinician may want to consider paroxetine for socially anxious children and adolescents. However, other SSRIs may also warrant consideration, especially in families where compliance is less than ideal, and withdrawal effects may emerge when a dose of paroxetine is missed. There is currently no clear evidence that one SSRI is superior to another in childhood anxiety. It is also not clear whether the combination of CBT and SSRI is better than either one alone, and whether this combination should be provided concurrently or sequentially. ${ }^{4}$ The risks and benefits of long-term SSRI use also warrant further investigation.

Katharina Manassis, MD, FRCP(C) Staff Psychiatrist, Hospital for Sick Children, Toronto; Associate Professor of Psychiatry, University of Toronto, Canada

1 Bernstein GA, Borchartdt CM, Perwien AR. Anxiety disorders in children and adolescents: A review of the past 10 years. J Am Acad Child Adolesc Psychiatry 1996;35:1110-19.

2 Kendall PC, Flannery-Schroeder E, Panichelli-Mindel SM, et al. Therapy for youths with anxiety disorders: A second randomized clinical trial. J Consult Clin Psychol 1997;65:366-80.

3 Research Unit on Pediatric Psychopharmacology Anxiety Study Group. Fluvoxamine for the treatment of anxiety disorders in children and adolescents. N Engl J Med 2001;344:1279-85.

4 Liashko V, Manassis K. Medicated anxious children: Characteristics and cognitive behavioral treatment response. Can J Psychiatry 2004;48:741-8. 